\title{
Mortalidad infantil y desigualdades sociales en Argentina, 1980-2017
}

\author{
Juan Carlos Bossio', Iván Sanchis², María Belén Herrero³, Gustavo Ariel Armando ${ }^{1}$ \\ y Sergio Javier Arias ${ }^{1}$
}

Forma de citar Bossio JC, Sanchis I, Herrero MB, Armando GA, Arias SJ. Mortalidad infantil y desigualdades sociales en Argentina, 1980-2017. Rev Panam Salud Publica. 2020;44:e127 https://doi.org/10.26633/RPSP.2020.127

RESUMEN

Objetivo. Estimar la tendencia de la mortalidad infantil, de la desigualdad entre jurisdicciones y de la desigualdad asociada a las condiciones sociales en Argentina entre 1980 y 2017.

Métodos. Estudio ecológico y de serie temporal de la mortalidad infantil y de su desigualdad. Se obtuvieron los datos oficiales de mortalidad infantil, de nacimientos y de necesidades básicas insatisfechas; se calculó la tasa de mortalidad infantil, el índice de Gini y el índice de concentración. También se analizó la tendencia con un modelo de regresión lineal y se calculó el coeficiente de regresión y su significación estadística. Resultados. La mortalidad infantil se redujo 71,2\% (de 32,41 a 9,34 por 1000 nacidos vivos). La desigualdad por jurisdicción también se redujo y el índice de Gini pasó de 0,163 a 0,09. La desigualdad asociada a las condiciones sociales también mostró una reducción, y el índice de concentración disminuyó de -0,153 a -0,079. Si bien la mortalidad infantil se redujo en todo el período, este descenso no siempre se acompañó de una reducción del índice de Gini y del índice de concentración.

Conclusiones. La tendencia de la tasa de mortalidad infantil fue al descenso mientras que la desigualdad en su distribución por jurisdicción y la desigualdad asociada a las condiciones sociales no siempre acompañaron esa reducción.

Palabras clave Mortalidad infantil; salud del niño; equidad en salud; inequidad social; estudios ecológicos; Argentina.

A pesar de los avances que se han observado en muchos de los indicadores generales de salud y de equidad, la desigualdad social sigue siendo una preocupación importante, especialmente para la salud materna e infantil.

Según las estimaciones de la Organización Mundial de la Salud (OMS), en 2018 murieron unos 5,3 millones de niños antes de cumplir cinco años (1). Esto significa que hubo más de 14.500 muertes de menores de cinco años por día. La muerte de la mayoría de estos niños ocurre por causas evitables, incluyendo enfermedades infecciosas para las cuales existe una cura, y se concentran en los países pobres $(1,2)$. La muerte de muchos niños a nivel global se vincula con la desigualdad geográfica de la mortalidad neonatal, infantil y en la niñez y con el hecho que, si bien en los últimos años estos indicadores se han reducido a

\footnotetext{
1 Instituto Nacional de Enfermedades Respiratorias "Emilio Coni", Santa Fe, Argentina.

2 Universidad Nacional del Litoral, Santa Fe, Argentina.
}

nivel global, su reducción no fue igual en todos los países (3). América Latina y el Caribe han avanzado en la reducción de la tasa de mortalidad infantil (TMI) y de la tasa de mortalidad neonatal (TMN). Entre 1990 y 2018, la TMI pasó de 43,3 por 1000 a 14,0 por 1000 y la TMN de 22,5 por 1000 a 9,1 por 1000 , lo que significó una mejora del $67,7 \%$ y 59,8 \%, respectivamente. A nivel mundial, para el mismo período, la reducción de estos indicadores fue menor: la TMI se redujo un 55,3\% y TMN un 51,7 \% (4). Sin embargo, en la región la distribución de estas muertes tampoco es uniforme. Factores como la desigualdad económica y el aislamiento geográfico de algunas zonas determinan enormes diferencias entre un país y otro (5). Por ejemplo, la TMI en Haití para 2018 fue 49,5 por 1000 , 13 veces más alta que la de Cuba (4). Pero las disparidades son grandes también 
dentro de un mismo país, donde un bebé que nace en condiciones de pobreza en algunas áreas tiene mayor probabilidad de perecer de manera prematura (6).

En Argentina, en el año 2017 se registraron 704609 nacidos vivos y fallecieron 6579 niños menores de 1 año, lo que determinó una TMI de 9,3 por cada mil nacidos vivos.

La mortalidad infantil en Argentina tuvo una tendencia al descenso en los últimos decenios, tanto en la mortalidad neonatal (hasta los 28 días), como en la post neonatal (4). Sin embargo, como ocurre a nivel mundial, la magnitud de la mortalidad infantil es muy desigual entre jurisdicciones (7).

El Ministerio de Salud de la Nación propuso que la reducción de la mortalidad infantil estuviera acompañada de una disminución de la desigualdad entre las jurisdicciones (7), en consonancia con la adaptación realizada a la adopción de los Objetivos para el Desarrollo Sostenible.

Numerosos estudios a nivel mundial han abordado la problemática de los múltiples y diversos factores que influyen en la desigual ocurrencia de la mortalidad infantil $(8,9)$. Las disparidades que se observan entre áreas geográficas y grupos de población no pueden ser simplemente atribuidas a las características y comportamientos de los individuos, ya que otros elementos relacionados con el área donde estos viven, las condiciones sociales y económicas, la infraestructura y la organización de las instituciones de salud juegan un papel clave y determinan en gran medida estas diferencias $(8,9)$. Asimismo, la salud y los resultados en salud se encuentran influenciados no sólo por las características del acceso y el uso de los servicios de salud, sino por una serie de factores de diversas dimensiones, incluidos los factores económicos y políticos, las condiciones de vida y las características del medioambiente $(10,11)$. Estos factores, que influyen directamente en el estado de salud, así como la interacción con factores biológicos, explican en gran medida las desigualdades de salud entre los diferentes grupos poblacionales (12).

Es preciso indagar la relación entre la ocurrencia de la muerte infantil con las desigualdades sociales, directamente vinculadas a las condiciones de vida (11). Comprender el comportamiento de la mortalidad infantil en un territorio, su tendencia en el tiempo y los factores que influyen en esto, es esencial para el establecimiento de acciones equitativas tendientes a disminuir las inequidades asociadas a la mortalidad infantil (13-15).

A través de los estudios ecológicos se ha buscado identificar, en las características sociales de la población y del área, las relaciones con la ocurrencia y distribución de las enfermedades y los resultados en salud (16-22). Una multiplicidad de estudios se ha llevado a cabo a nivel mundial con el objetivo de determinar la desigualdad en la mortalidad infantil entre jurisdicciones y su relación con las condiciones de vida $(8,9,11,14-15,23)$. Sin embargo, no hemos encontrado en Argentina estudios que adopten esta perspectiva, para comprender la tendencia de la mortalidad, y la desigual distribución asociada a las condiciones sociales.

El objetivo de este estudio es estimar la tendencia de la mortalidad infantil en Argentina entre 1980 y 2017, y los cambios que acompañaron esa tendencia en la desigualdad y en la desigualdad asociada a las condiciones sociales entre jurisdicciones.

\section{MATERIALES Y MÉTODOS}

Se realizó un estudio ecológico espacial y de serie temporal, con las 24 jurisdicciones del país como unidades de análisis. Se analizaron todas las muertes infantiles en el período 1980 - 2017, y la población de estudio correspondió a todos los nacidos vivos y muertes menores de un año entre 1980 y 2017; y la población total y en hogares con necesidades básicas insatisfechas para los años censales 1980, 1991, 2001 y 2010.

Se solicitaron las bases de datos de mortalidad de menores de un año y de nacidos vivos de la Dirección de Estadísticas e Información de Salud del Ministerio de Salud de la Nación, correspondientes al período 1980-2017, por jurisdicción (24 provincias).

Se obtuvo asimismo la población con base en la información oficial del Instituto Nacional de Estadística y Censos (INDEC) y se empleó el porcentaje de población en hogares con necesidades básicas insatisfechas (NBI) para el total del país, por jurisdicción (para todos los años censales), del INDEC, como indicador de la condición social en cada jurisdicción.

Se calculó la TMI por 1000 nacidos vivos para todo el período 1980-2017, para el total del país y sus jurisdicciones.

Para analizar la desigualdad en la distribución de la mortalidad infantil por jurisdicción, se calculó el índice de Gini (IG) para cada año del período 1980-2017; y el índice de concentración (IC) para evaluar la desigualdad asociada a las desigualdades sociales para los años 1980, 1991, 2001 y 2010; ambos con su intervalo de confianza del 95\%.

Se utilizó el porcentaje de población en hogares con NBI como indicador de condiciones de vida. Este indicador es elaborado por el INDEC en cada año censal e incluye un conjunto de variables de las condiciones de vida de la población considerando no solamente la insuficiencia de ingresos (24). Incorpora otras condiciones como, por ejemplo, características de la vivienda, nivel de instrucción de los integrantes de la familia y condiciones de hacinamiento, que permiten una medición más amplia de la pobreza, asociada a las privaciones materiales anteriores (24).

A continuación, se analizó la tendencia de la TMI y la del IG para la desigualdad entre jurisdicciones con un modelo de regresión lineal y se calculó el coeficiente de regresión y su significación estadística.

Se analizó la existencia de puntos de quiebre de las tendencias mediante el programa JoinPoint y, para cada período identificado se calculó el coeficiente de regresión, que se expresó como Variación Anual Promedio (VAP) porcentual, con su intervalo de confianza del 95\% y su significación estadística (25).

La tendencia de la desigualdad asociada a las condiciones sociales se expresó como variación porcentual en cada período.

Para analizar el comportamiento de las provincias que acompañó a los cambios en el IG, se las agrupó en cuartiles, según su TMI en el año identificado como punto de quiebre. Se analizó luego la tendencia de cada uno de los cuartiles durante el período delimitado por los puntos de quiebre, con un modelo de regresión lineal y se calculó la VAP de cada cuartil con su intervalo de confianza del 95\% y su significación estadística.

El mismo procedimiento se utilizó para analizar el comportamiento de las provincias que acompañó a los cambios en el IC, agrupando a las provincias en cuartiles, según su porcentaje de población en hogares con NBI en los años para los cuales se obtuvo el indicador (1980, 1991, 2001 y 2010), y realizando el análisis de la tendencia de los cuartiles en cada período. También se utilizó un modelo de regresión lineal y se calculó la variación anual promedio VAP de cada cuartil con su intervalo de confianza del 95\% y su significación estadística. 
Para los extremos de los períodos identificados, se calculó la media de la TMI por jurisdicción con su intervalo de confianza mediante bootstrap y se compararon las medias mediante el método de las permutaciones. Para todos los análisis se utilizó un nivel de confianza del $95 \%$ y para el procesamiento y análisis de la información se utilizó el paquete estadístico $\mathrm{R}$ y el Joinpoint 4.2.0.2.

Este estudio se realizó utilizando datos anonimizados por lo que cumple con las condiciones adecuadas para prescindir de evaluación por un comité de ética, según lo establecido por el Ministerio de Salud de la Nación de la República Argentina (26).

\section{RESULTADOS}

La mortalidad infantil en Argentina se redujo 71,2\% entre 1980 y 2017, pasando de 32,41 por 1000 nacidos vivos a 9,34 por 1000 nacidos vivos (cuadro 1 ).

Durante el mismo período, la desigualdad en la distribución de la mortalidad infantil por jurisdicción tuvo una reducción menor: 44,8\%. El IG pasó de 0,163 (IC 0,116; 0,210) a 0,09 (IC 0,047; 0,133) (cuadro 1).

La desigualdad asociada a las condiciones sociales también mostró una reducción entre 1980 y 2010, primer y último año

CUADRO 1. Tendencia de la mortalidad infantil, de la desigualdad en la distribución y de la desigualdad asociada a las condiciones sociales, por jurisdicción. Argentina, 1980-2017

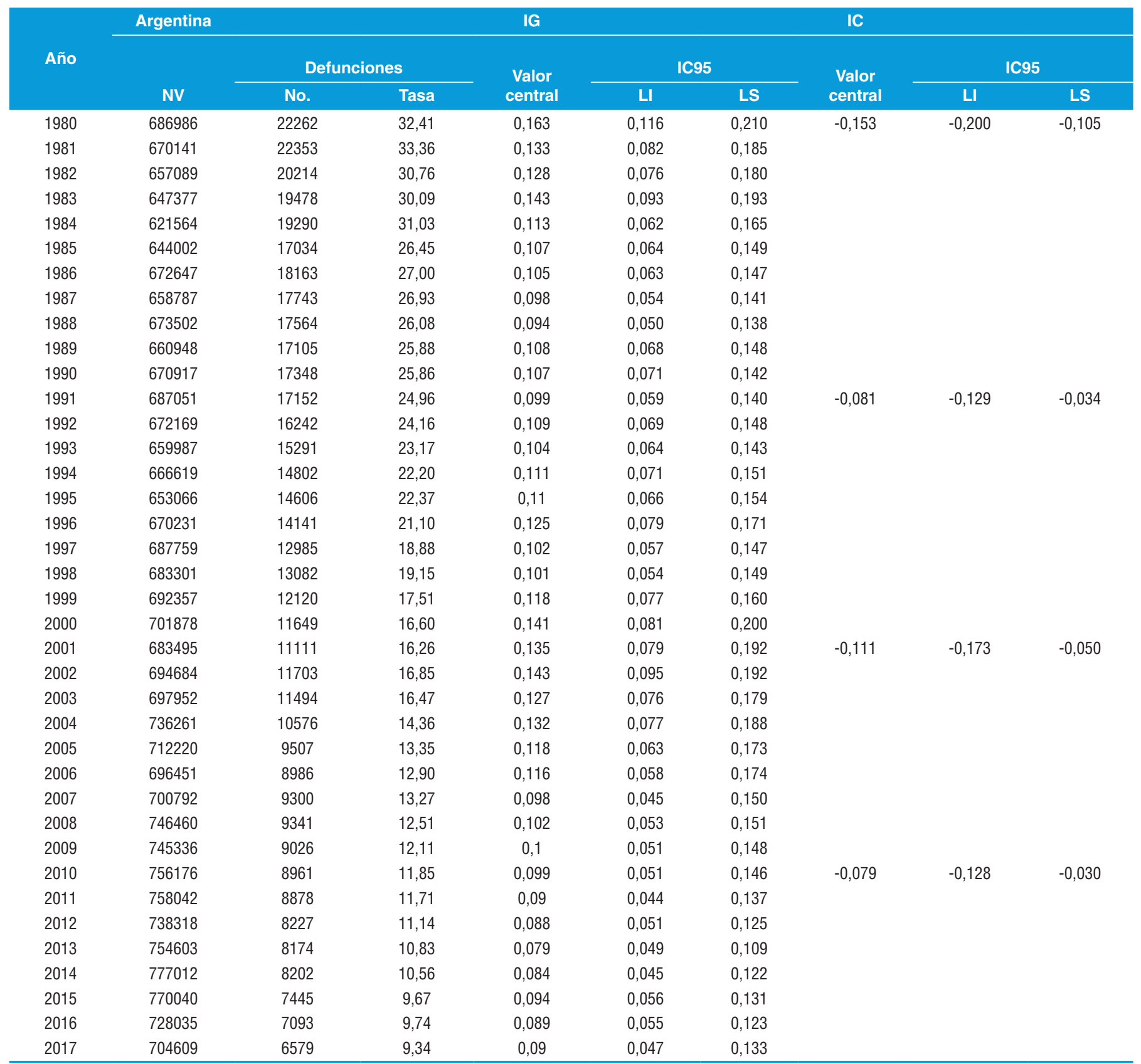

Fuente: Elaboración propia, con base en datos de la Dirección de Estadísticas e Información de Salud (DEIS), Ministerio de Salud de la Nación. Argentina, 2019.

IG: İndice de Gini; IC: Índice de concentración; NV: Nacidos vivos; IC95: Intervalo de confianza del 95 \%; LI: Límite inferior; LS: Límite superior 
censal del período. El IC se redujo $48,0 \%$, pasando de $-0,153$ (IC $-0,200 ;-0,105$ ) a $-0,079$ (IC $-0,128 ;-0,030$ ) (cuadro 1 ).

La reducción de la TMI y de la desigualdad no fue continua a lo largo del período (figura 1).

La TMI tuvo una tendencia al descenso $(p<0,0001)$ con una variación anual promedio de $-3,48 \%$ (IC -3,62; -3,35). El descenso no fue estable y se pueden identificar dos puntos de quiebre en 1993 y 2006 ( $p<0,05$ ), que diferencian tres períodos, todos con tendencia al descenso.

La tendencia de la TMI entre los puntos de quiebre, muestra que el descenso más rápido se observó en el período 1993 - 2006, con una VAP de $-4,31 \%$ (IC -4,88; -3,73). El descenso más lento se dio entre 1980 y 1993, con una VAP de -2,56\% (IC -3,05; -2,07). En el último período (2006 - 2017), la VAP fue -3,15\% (IC -3,54;-2,76).

El descenso de la TMI no siempre se acompañó de una reducción en la desigualdad entre las jurisdicciones (figura 1). La desigualdad, medida con el IG, mostró una reducción de 44,8\% en todo el período, con una VAP de - $0,84 \%$ (IC -1,27; - 0,$40 ; \mathrm{p}=$ $0,0004)$; pero se pueden identificar tres puntos de quiebre $(\mathrm{p}<$ $0,05)$ que determinan cuatro períodos diferentes en la tendencia de la desigualdad.

Entre 1980 y 1987, la desigualdad se redujo 39,9\%, con una VAP para el IG de $-6,41 \%(-8,93 ;-3,83 ; p=0,0010)$. En cambio, a partir de 1987 y hasta 2002, la desigualdad aumentó 45,9\% con una VAP del IG de 2,20\% $(1,21 ; 3,20 ; \mathrm{p}=0,0003)$, alcanzando el valor de 0,143 .

Desde 2002 y hasta 2013, la tendencia de la desigualdad fue nuevamente al descenso. El IG se redujo $44,8 \%$ con una VAP de $-4,73 \%(-5,55 ;-3,91 ; \mathrm{p}<0,0001)$. A partir de 2013 y hasta 2017 , la desigualdad aumentó $13,9 \%$, y la VAP del IG fue 1,27\%, no estadísticamente significativa ( $\mathrm{p}=0,6263)$. El valor más alto de este período se registró en el año 2015, 19,0\% mayor que el de 2013 (cuadro 1).

La tendencia de la TMI de las provincias, agrupadas en cuartiles según su valor al inicio de cada uno de los períodos identificados para el IG, o agrupadas en cuartiles según el valor de su indicador social al inicio de los períodos identificados para el IC, fue diferente en cada período (figuras 2 y 3 ).

Durante los dos períodos en que la desigualdad, medida mediante el IG, se redujo (1980-1987 y 2002-2013), las provincias con peor situación (TMI > 35 por 1000$)$ tuvieron un descenso de la TMI mayor al de las provincias con mejor situación (TMI $<34$ por 1000). Como resultado, la razón de la tasa del cuartil superior (TMI > 43 por 1000) versus el cuartil inferior $(\mathrm{TMI}<29$ por 1 000) pasó de 1,95 a 1,39 en el período 1980 - 1987 y de 2,14 a 1,35 en el período $2002-2013$.

FIGURA 1. Comparación de la tendencia de la tasa de mortalidad infantil (TMI) y de la desigualdad en su distribución por jurisdicciones. Tasas por 1000 nacidos vivos e índice de Gini (IG). Argentina, 1980-2017

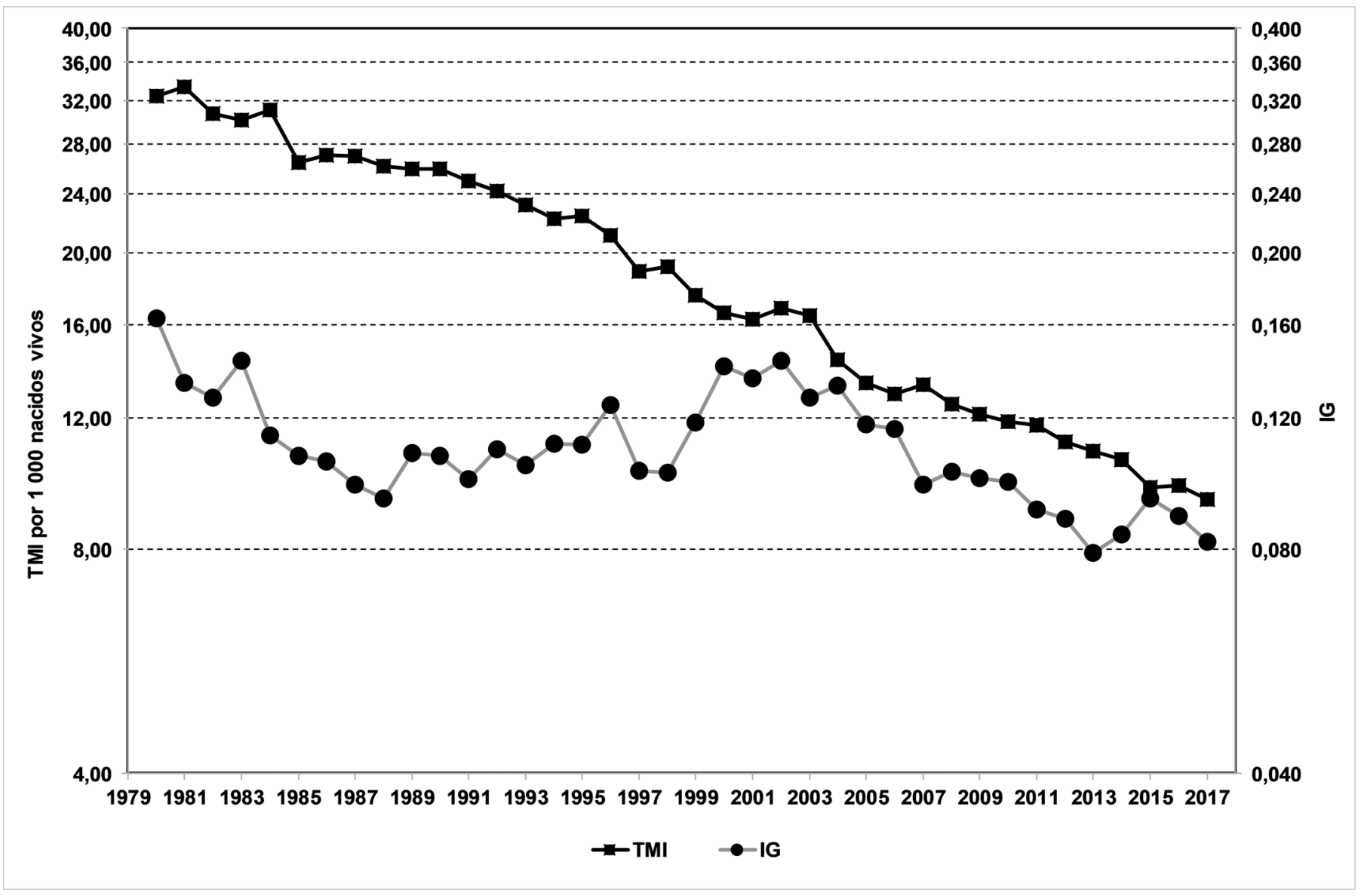

Fuente: Elaboración propia, con base en datos de la Dirección de Estadísticas e Información de Salud (DEIS), Ministerio de Salud de la Nación. Argentina, 2019. 
FIGURA 2. Variación Anual Promedio (VAP) porcentual de la tasa de mortalidad infantil (TMI) en Argentina, por jurisdicciones agrupadas en cuartiles según su valor al inicio de cada período

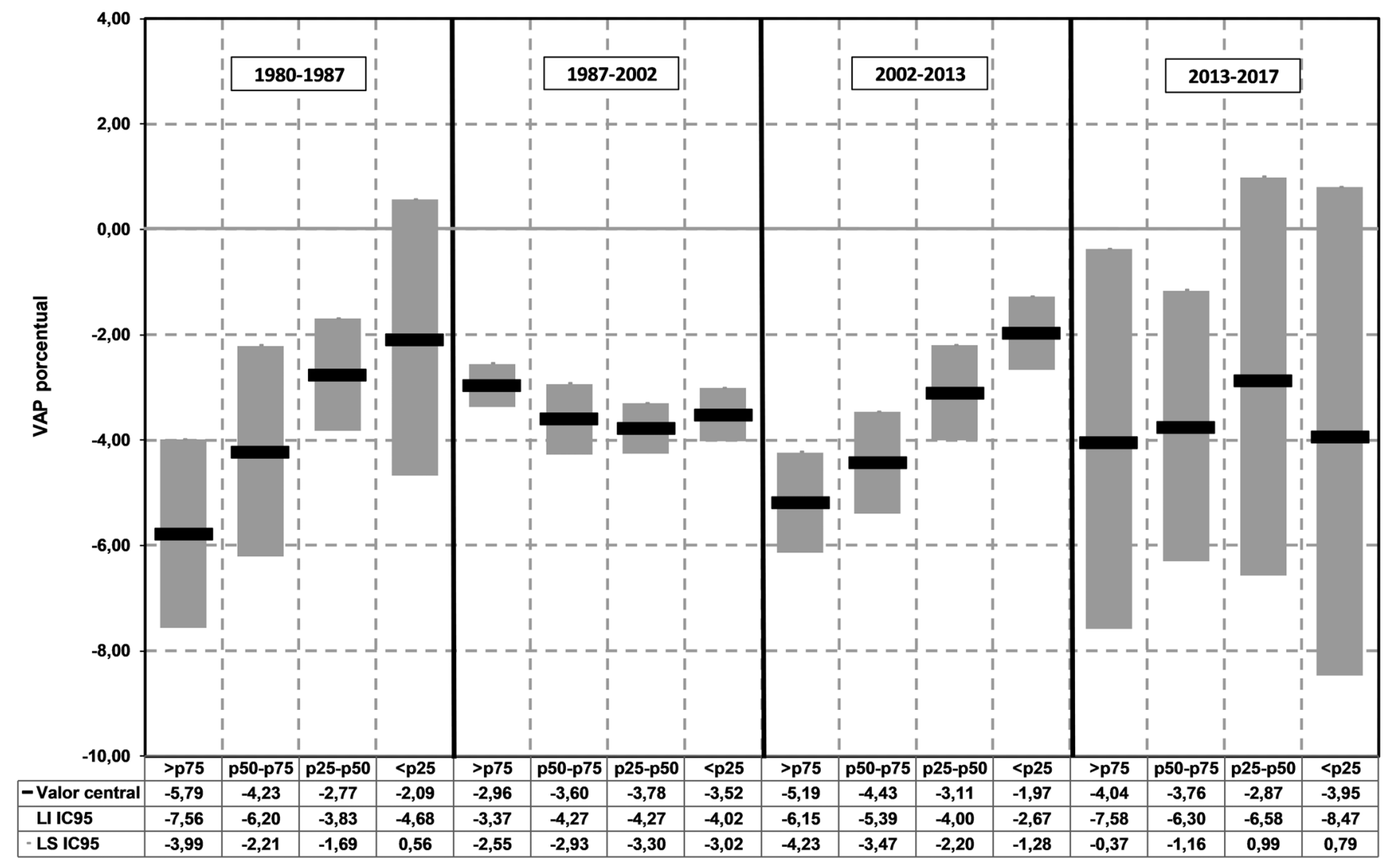

Fuente: Elaboración propia, con base en datos de la Dirección de Estadísticas e Información de Salud (DEIS), Ministerio de Salud de la Nación. Argentina, 2019. LI IC95: Límite inferior del intervalo de confianza del $95 \%$; LS IC95: Límite superior del intervalo de confianza del $95 \%$.

En cambio, cuando la desigualdad aumentó (períodos 1987 2002 y 2013 - 2017), las tendencias al descenso de los cuartiles fueron similares. La razón de la tasa del cuartil superior e inferior pasó de 1,69 a 1,84 en el primer período y de 1,54 a 1,59 en el segundo, mostrando un aumento en ambos casos.

El descenso de la desigualdad asociada a las condiciones sociales, medida a través del IC, tampoco fue uniforme durante todo el período (cuadro 1). Entre 1980 y 1991, el IC se redujo $46,62 \%$; a partir de ese año y hasta 2001, se incrementó $36,62 \%$ y volvió a disminuir 28,69\% hasta 2010.

Agrupando las jurisdicciones según cuartiles de acuerdo con el valor del indicador de condición social, entre 1980 y 1991, cuando el IC se redujo, las provincias que integraron los cuartiles con peor situación social (NBI > $38 \%$ ) tuvieron una reducción similar y mayor a la del cuartil con mejor situación social (NBI < $22 \%$ ) (figura 3).

En cambio, entre 1991 y 2001, los mayores descensos se observaron en los cuartiles con peor y mejor condición social (NBI $>31 \%$ y NBI $<14 \%$, respectivamente). Los cuartiles intermedios, en cambio, tuvieron una reducción similar a la del período previo.

A partir de 2001 y hasta 2010, las provincias que mostraron un descenso mayor fueron las que integraron el cuartil p50 - p75. Respecto al período precedente, las provincias en peor situación social tuvieron un descenso de la desigualdad asociada a las condiciones sociales similar, mientras que las provincias en mejor situación social tuvieron un descenso menor.

\section{DISCUSIÓN}

Los resultados de nuestro estudio muestran que la TMI en Argentina tuvo una tendencia sostenida al descenso durante el período estudiado, tal como se observó tanto a nivel global como a nivel local en numerosos países $(27,28)$. Los hallazgos mostraron un descenso total de 71,2\% en el período 1980-2017. Este valor resulta mayor al observado a nivel global (29) y similar al observado en otros estudios realizados para la región latinoamericana. En la ciudad de Pelotas, Brasil, se registró un $62,0 \%$ de descenso de la TMI para un período de 33 años (19822015), según lo reportado por Menezes y cols. (30). En Chile, la reducción fue mayor: en el período 1970-2003, durante el cual la TMI se redujo un 90,1\%, según reportaron Kaempffer y Medina (31). Uruguay también registró un descenso mayor al de Argentina, logrando una reducción de 53\% durante el período 1990-2009 (32).

Nuestro estudio también mostró que el descenso de la TMI no siempre se acompañó de una reducción de la desigualdad entre las jurisdicciones ni de la desigualdad asociada a las 
FIGURA 3. Variación Anual Promedio (VAP) porcentual de la tasa de mortalidad infantil en Argentina, por jurisdicciones agrupadas en cuartiles según el valor del índice de necesidades básicas insatisfechas al inicio de cada período

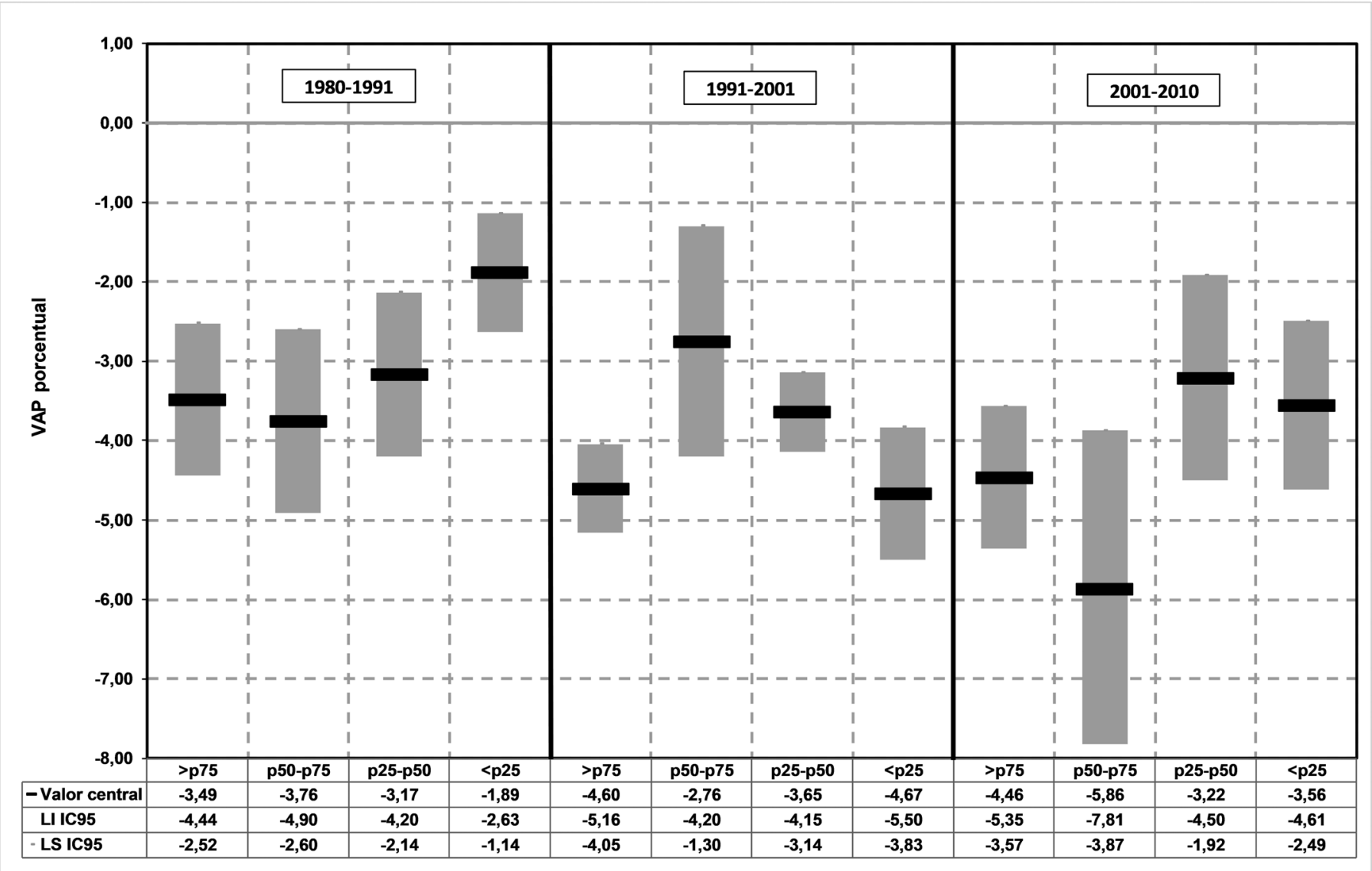

Fuente: Elaboración propia, con base en datos de la Dirección de Estadísticas e Información de Salud (DEIS), Ministerio de Salud de la Nación. Argentina, 2019. LI IC95: Límite inferior del intervalo de confianza del 95\%; LS IC95: Límite superior del intervalo de confianza del $95 \%$.

condiciones sociales de la población. Esto se observó tanto comparando la tendencia de la TMI con la del indicador de desigualdad (IG) como desagregando la tendencia por cuartiles según el nivel de su TMI al inicio de cada período.

La falta de disminución de la desigualdad a pesar del descenso continuo de la TMI ha sido también documentada en otros países tanto de Latinoamérica $(33,34)$ como de otras regiones (35). En la mayoría de estos estudios los autores destacan la importancia de acompañar el análisis de la tendencia de la TMI con un análisis de la tendencia de la desigualdad de este indicador entre áreas geográficas y grupos poblacionales.

Diferencias en la tendencia de la desigualdad al desagregarla por cuartiles u otras características de la población incluyendo diferencias en las condiciones sociales también han sido encontradas en otros estudios. Schneider y cols. (33) observaron un comportamiento distinto de la tendencia de la TMI entre grupos con diferente producto nacional bruto, pero que resultó en una razón de tasa similar entre los quintiles extremos en el período de estudio (33). Simeoni y cols. (35), en un reciente estudio, compararon las tendencias de grupos según la condición de inmigrantes o no inmigrantes y áreas geográficas; y observaron que la brecha entre las regiones tuvo una tendencia estable en el período estudiado (35).
Nuestro estudio muestra que la disminución de la desigualdad en el primer y último período se produjo a expensas de una mayor tendencia al descenso en la TMI de las provincias que integraban el cuartil con las tasas más elevadas, en comparación con aquellas que integraban el cuartil con las tasas más bajas. Sin embargo, esto no se observó en el período en el que la desigualdad aumentó. En este último, pudo observarse que el descenso de las provincias fue similar, es decir, independiente del cuartil que integraban al inicio del período o bien que, incluso, tuvieron un descenso menor aquellas en los cuartiles en peor situación.

De manera similar, los resultados muestran que, en los dos períodos de descenso de la desigualdad asociada a las condiciones sociales, la reducción de la mortalidad infantil fue mayor en los cuartiles con peor situación social que, en aquellos con mejor situación social.

Este estudio se limita a describir la evolución de la mortalidad infantil en Argentina durante las últimas décadas y de la desigualdad en su distribución en las jurisdicciones, tanto en función de su magnitud como de las condiciones sociales de su población. De este modo, no permite establecer otras posibles características, tanto de la población como de las áreas de análisis, que se asocien al comportamiento del indicador. Además, 
por tratarse de un estudio ecológico, la relación entre la evolución de la TMI y su distribución asociada a las condiciones sociales se analiza sólo a nivel de grupo (jurisdicciones), lo que no permite establecer conclusiones sobre el impacto de esas condiciones a nivel individual. Las disparidades que se observan entre áreas geográficas y grupos de población se deben a múltiples factores, y no pueden ser simplemente atribuidas a las características y comportamientos de los individuos, ya que otros elementos relacionados con el área donde estos viven, las condiciones sociales y económicas, la infraestructura y la organización de las instituciones de salud juegan un papel clave y determinan en gran medida estas diferencias (28). En este sentido, el análisis de las posibles causas de que el comportamiento haya sido diferente en los tres períodos (que sería análogo a posibles causas de la existencia de estos períodos en primer lugar, es decir, a la existencia de puntos de quiebre de la tendencia), escapa a los objetivos del trabajo.

\section{CONCLUSIONES}

La tendencia de la TMI en Argentina en el período de estudio fue al descenso mientras que la desigualdad en su distribución por jurisdicción y la desigualdad asociada a las condiciones sociales acompañaron esa tendencia en dos períodos, pero aumentaron entre ellos. Intervenciones específicas dirigidas a las áreas de mayor TMI o NBI deberían ponerse en práctica para garantizar que la disminución de la TMI se acompañe siempre de una reducción de la inequidad.

Contribución de los autores. JCB, IS, MBH concibieron el estudio original y escribieron el manuscrito. Todos los autores analizaron e interpretaron los datos y resultados, revisaron el manuscrito y aprobaron la versión final.

Agradecimientos. Los autores agradecen a la Dirección de Estadísticas e Información en Salud (DEIS) del Ministerio de Salud de la Nación, Argentina, por la provisión de los datos que hicieron posible este estudio.

Conflictos de intereses. Ninguno declarado por los autores.

Declaración. Las opiniones expresadas en este manuscrito son responsabilidad del autor y no reflejan necesariamente los criterios ni la política de la RPSP/PAJPH y / o de la OPS.

\section{REFERENCIAS}

1. World Health Organization. Children: reducing mortality. Ginebra: WHO, 2019. https://www.who.int/news-room/fact-sheets/ detail/children-reducing-mortality. Acceso el 23 de abril 2020

2. Chao F, You D, Pedersen J, Hug L, Alkema L. National and regional under- 5 mortality rate by economic status for low-income and middle-income countries: a systematic assessment. Lancet Glob Health. 2018 May;6(5):e535-e547

3. Burstein, R., Henry, N.J., Collison, M.L. et al. Mapping 123 million neonatal, infant and child deaths between 2000 and 2017. Nature. 2019; 574, 353-358

4. United Nations Inter-Agency Group for Child Mortality Estimation (UN IGME). Child Mortality Estimates. New York: UN IGME; 2019. Disponible en: https://childmortality.org/2018/08/17/datacompilation/ Acceso el 23 de abril de 2020

5. United Nations International Children's Emergency Fund. Health Equity Report 2016: Analysis of reproductive, maternal, newborn, child and adolescent health inequities in Latin America and the Caribbean to inform policymaking. New York: UNICEF; 2016. Disponible en: https://reliefweb.int/sites/reliefweb.int/files/ resources/20160906_UNICEF_HealthEquityReport.pdf. Acceso el 23 de abril de 2020

6. Bachelet M. Data on child deaths are a call for justice. Nature. 2019;Oct;574(7778):297

7. Dirección Nacional de Maternidad e Infancia. Análisis de la Mortalidad Materno Infantil. República Argentina, 2003-2012. Ministerio de Salud de la Nación, Argentina. 2012;1:298

8. Mosley WH, Chen LC. An analytical framework for the study of child survival in developing countries. 1984. Bull World Health Organ. 2003; 81(2): 140-145Mosley WH, Chen LC. An analytical framework for the study of child survival in developing countries. In: Mosley WH \& Chen LC. Child survival: strategies for research. Pop Devel Rev. 1984;10:25-45

9. Victora CG, César JA. Saúde materno-infantil no Brasil - Padrões de morbimortalidade e possíveis intervenções. Rio de Janeiro: MEDSI. 2003:415-467

10. Genowska A, Jamiolkowski J, Szafraniec K, Stepaniak U, Szpak A, Pajak A. Environmental and socio-economic determinants of infant mortality in Poland: an ecological study. Environ Health. 2015; 14: 61

11. Huda TM, Tahsina T, El Arifeen S, Dibley MJ. The importance of intersectoral factors in promoting equity-oriented universal health coverage: a multilevel analysis of social determinants affecting neonatal infant and under-five mortality in Bangladesh. Glob Health Action. 2016;9:29741

12. Marmot M. Social determinants of health inequalities. Lancet. 2005; 365: 1099_104

13. Guimarães MJB, Marques NM, Mello Filho DA, Szwarcwald CL. Condição de vida e mortalidade infantil: diferenciais intra-urbanos no Recife, Pernambuco, Brasil. Cad Saúde Pública. 2003; 19(5):1413-1424

14. Morais Neto OL, Barros MBA. Fatores de risco para mortalidade neonatal e pósneonatal na Região Centro-Oeste do Brasil: linkage entre bancos de dados de nascidos vivos e óbitos infantis. Cad de Saúde Pública. 2000; 16(2):477-485

15. Almeida $S$, Barros MBA. Atenção à saúde e mortalidade neonatal: estudo caso controle realizado em Campinas, São Paulo. Rev Br Epid. 2004;7(1):22-35

16. Krieger N. Overcoming the absence of socioeconomic data in medical records: validation and application of a census-based methodology. Am J Public Health. 1992;82:703-10

17. Krieger N, Chen JT, Waterman PD et al. Geocoding and Monitoring of US Socioeconomic Inequalities in Mortality and Cancer Incidence: Does the Choice of Area-based Measure and Geographic Level Matter? The Public Health Disparities Geocoding Project. Am J of Epidemiol. 2002; 156(5):471-482

18. Diez Roux AV. Conceptual approaches to the study of health disparities. Annu Rev Public Health. 2012;33:41-58.

19. Hoffmann R, Borsboom G, Saez M et al. Social differences in avoidable mortality between small areas of 15 European cities: an ecological study. Int J Health Geogr. 2014;13:8.

20. Cerda M, Morenoff JD, Hansen BB et al. Reducing violence by transforming neighborhoods: a natural experiment in Medellin, Colombia. Am J Epidemiol. 2012;175(10):1045-1053

21. Chetty R, Stepner M, Abraham S et al. The Association Between Income and Life Expectancy in the United States, 2001-2014. JAMA. 2016;315(16):1750-1766.

22. Caicedo-Velásquez B, Álvarez-Castaño LS, Marí-Dell'Olmo M, Borrell C. Evolución de las inequidades en mortalidad por causas externas entre los municipios de Antioquia (Colombia). Gac Sanit. 2016;30(4): 279-286

23. Herrero MB, Bossio JC. Determinantes sociales de la mortalidad infantil por causas reducibles en la Argentina, 2009-2011. Población y Salud en Mesoamérica. 2017;15(1):1-29 
24. Instituto Nacional de Estadística y Censo (INDEC). Necesidades básicas insatisfechas. Buenos Aires: INDEC., 2020. Disponible en: https:/ / www.indec.gob.ar/indec/web/Nivel4-Tema-4-47-156. Acceso el 23 de abril de 2020

25. Organización Panamericana de la Salud. Lineamientos básicos para el análisis de la mortalidad. Washington, DC: OPS, 2017. Disponible en : https://iris.paho.org/handle/10665.2/34492. Acceso el 23 de abril de 2020

26. Ministerio de Salud de la Nación, Argentina. Guía para investigaciones en Salud Humana. Buenos Aires: Ministerio de Salud, 2011. Disponible en: http://www.msal.gob.ar/images/stories/bes/ graficos/0000001274cnt-guia_investigaciones_salud_humana.pdf. Acceso el 23 de abril de 2020

27. United Nations Inter-Agency Group for Child Mortality Estimation (UN IGME). Levels \& Trends in Child Mortality: Report 2019. Estimates developed by the UN Inter-agency Group for Child Mortality Estimation. New York: UN IGME; 2019. Disponible en: https:// www.unicef.org/reports/levels-and-trends-child-mortality-report-2019 Acceso el 23 de abril de 2020

28. Finkelstein JZ et al. Trend in infant mortality rate in Argentina within the framework of the Millennium Development Goals. Arch Argent Pediatr 2016;114(3):216-222

29. World Health Organization. Infant mortality. Situation and trends. Ginebra: WHO, 2017. Disponible en: https://www.who.int/gho/ child_health/mortality/neonatal_infant_text/en/ Acceso el 23 de abril de 2020 .
30. Menezes AMB, Barros FC, Horta BL et al. Stillbirth, newborn and infant mortality: trends and inequalities in four population-based birth cohorts in Pelotas, Brazil, 1982-2015. Int J Epidemiol. 2019; 48(1):i54-i62

31. Kaempffer AR, Medina EL. Mortalidad infantil reciente en Chile: Éxitos y desafíos. Rev chil pediatr. 2006;77(5):492-500

32. Giachetto G. Mortalidad infantil en Uruguay: una mirada crítica. Arch Pediatr Urug. 2010;81(3):139-140

33. Schneider MC, Castillo-Salgado $C$ et al. Trends in infant mortality inequalities in the Americas: 1955-1995. J Epidemiol Community Health. 2002;56(7):538-41

34. Warren Hertel-Fernandez A, Giusti AE, Sotelo JM. The Chilean infant mortality decline: improvement for whom? Socioeconomic and geographic inequalities in infant mortality, 1990-2005. Bull World Health Organ. 2007;85:733-820

35. Simeoni S, Frova L, De Curtis M. Inequalities in infant mortality in Italy. Ital J Pediatr. 2019; 45:11.

Manuscrito recibido el 16 de diciembre de 2019. Aceptado para su publicación, tras revisión, el 1 de mayo de 2020

\section{Infant mortality and social inequalities in Argentina, 1980-2017}

ABSTRACT

Keywords
Objective. To estimate the trend in infant mortality, inequality between jurisdictions and inequality associated with social conditions in Argentina between 1980 and 2017

Methods. Ecological and time series study of infant mortality and its inequality. Official data on infant mortality, births and unmet basic needs were obtained; the infant mortality rate, the Gini index and the concentration index were calculated. The trend was also analyzed with a linear regression model and the regression coefficient and its statistical significance were calculated.

Results. Infant mortality was reduced by $71.2 \%$ (from 32.41 to 9.34 per 1000 live births). Inequality by jurisdiction also decreased, and the Gini index fell from 0,163 to 0,09. Inequality associated with social conditions also showed a reduction, and the concentration index was reduced from -0.153 to -0.079 . Although infant mortality declined throughout the period, this decline was not always accompanied by a reduction in the Gini index and the concentration index.

Conclusions. The trend in the infant mortality rate decreased while the inequality in its distribution by jurisdiction and the inequality associated with social conditions did not always accompany this reduction.

Infant mortality; child health; health equity; social inequity; ecological studies; Argentina. 